\title{
Laparoscopic Nissen fundoplication in the treatment of Barrett's esophagus - 10 years of experience
}

\author{
Marcin Migaczewski, Michał Pędziwiatr, Maciej Matłok, Andrzej Budzyński \\ Second Department of General Surgery, Jagiellonian University Medical College, Krakow, Poland
}

Videosurgery Miniinv 2013; 8 (2): 139-145

DOI: 10.5114/wiitm.2011.32941

\begin{abstract}
Introduction: Barrett's esophagus (BE) is a state in which the distal portion of esophageal mucosa becomes lined with cylindrical epithelium as a result of adaptive remodeling. It is widely accepted that the metaplastic lesions result from chronic irritation with gastric and/or duodenal contents in the course of reflux disease. For many years, research centered on the risk factors of BE and resulting adenocarcinoma. Anti-reflux operations are the only procedures which offer the possibility of treating the cause by restoring the anatomic barrier responsible for guarding against irritating effects of gastroduodenal content on the distal esophagus. Total (i.e. $360^{\circ}$ ) laparoscopic Nissen fundoplication (LNF) is considered the most effective amongst these procedures. Still, controversies related to the indications for anti-reflux surgery are frequently encountered.

Aim: Retrospective analysis of long-term treatment outcomes in patients with BE subjected to laparoscopic Nissen fundoplication.

Material and methods: The group included 42 BE patients, amongst them 30 men and 12 women. Initially, all the patients were treated conservatively for at least 1 year. The subgroup with dysplasia was subjected to preoperative argon plasma coagulation (APC). From 1 year after surgery (laparoscopic Nissen fundoplication), control biopsy specimens were obtained from the gastroesophageal junction of all the patients.

Results: None of the patients showed the development of esophageal adenocarcinoma during the follow-up period. Furthermore, no cases of dysplasia progression or de novo development of dysplasia were observed in the analyzed group. In the initial 12-24 months after surgery, complete regression of metaplasia was documented in 7 (31.8\%) patients from group $A$, and a reduction in the area of Barrett's metaplasia was observed in another 7 patients (31.8\%). Throughout the period of this study, persistent planoepithelial re-epithelialization was observed in 14 (70\%) group B patients, i.e. in individuals with baseline dysplasia subjected to preoperative argon plasma ablation. In the remaining patients of this group, the developed changes of BE character were less advanced than at baseline.

Conclusions: Our opinion is that laparoscopic Nissen fundoplication, as a result of high effectiveness, represents the method of choice in the treatment of BE in the case of patients who were qualified for surgery.
\end{abstract}

Key words: laparoscopy, Nissen fundoplication, Barrett's esophagus.

\section{Introduction}

Barrett's esophagus (BE) is a state in which the distal portion of the esophageal mucosa becomes lined with cylindrical epithelium as a result of adaptive remodeling. The replacement of normal squamous epithelium with glandular epithelium can be observed on microscopic examination (Photo 1). It is widely ac- 


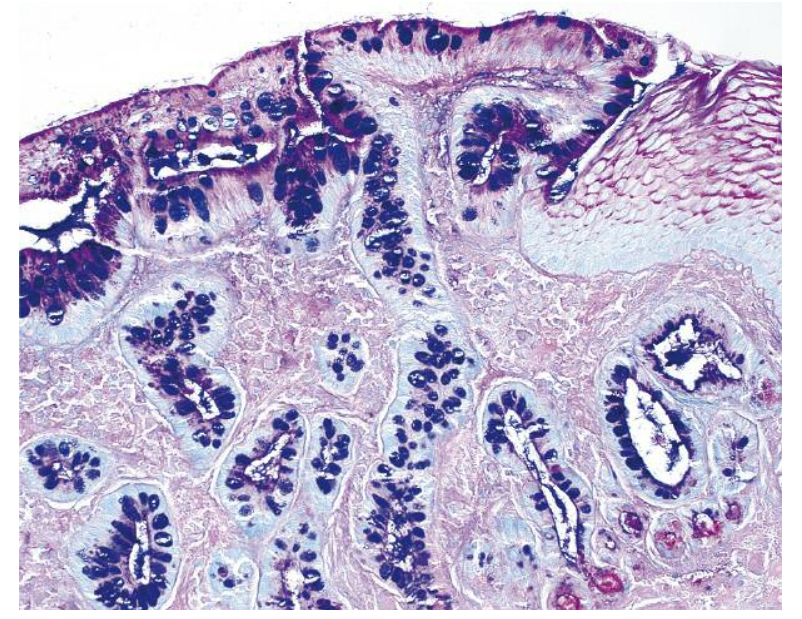

Photo 1. Barrett's metaplasia - the glandular epithelium, intestinalisation without dysplasia. Histochemical staining Alcian blue + PAS $(\mathrm{pH}$ 2.5) - acidic mucus tinged with blue, neutral red, pink, 20x lens (courtesy of Dr. K. Urbańczyk Department of Pathology, Jagiellonian University Medical College)

cepted that the metaplastic lesions result from chronic irritation with gastric and/or duodenal contents in the course of reflux disease. Also, as a result of adaptive remodeling of the esophagus, the $Z$ line does not correspond to the upper threshold of gastric folds.

Many authors consider BE as the most advanced stage of gastroesophageal reflux disease (GERD). Although the hypothesis on the close association between glandular metaplasia of the gastroesophageal junction and the risk of esophageal adenocarcinoma is widely accepted, the exact mechanisms behind this relationship are not fully understood. In view of the discrepancies with regards to the possible clinical picture, frequent modifications of disease definition, and poorly understood pathogenic mechanisms of $B E$, a unified diagnostic and therapeutic algorithm has not been developed thus far for this group of patients [1].

Control of duodenogastroesophageal reflux constitutes the principal therapeutic objective in BE patients [2]. Anti-secretive pharmacotherapy, mostly with proton pump inhibitors (PPIs), is of vital importance. However, according to numerous authorities, commonly used PPIs only attenuate the symptoms of reflux, but do not completely normalize $\mathrm{pH}$ of the distal esophagus [2]. Additionally, such negative consequences of chronic PPI administration as hypergastrinemia, and disturbances of the bacterial gastric microflora, potentially promoting the development of some malignancies, should not be underestimated. Even assuming that PPIs effectively reduce gastric secretion of acids, decidedly, they do not play an important role in the case of non-acidic reflux. Moreover, it should be emphasized that bile acid salts, the principal components of alkaline refluxate, play a particularly important role in the promotion of esophageal adenocarcinoma development [3-5].

For many years, research centered on the risk factors of BE and resulting adenocarcinoma. Although the role of chronic irritation of the distal esophageal mucosa with refluxate is unquestionable, still it remains unexplained why some patients develop solely mucosal inflammation, while Barrett's metaplasia is observed in others. According to the literature, the risk factors of $B E$ include duration of exposure and the composition of the refluxate. Furthermore, a number of authors point out that the effective suppression of gastric secretion not only leads to planoepithelial re-epithelialization, but is also reflected by reduced expression of certain proliferation-specific biomarkers [6].

Anti-reflux operations are the only procedures which offer the possibility of treating the cause by restoring the anatomic barrier responsible for guarding against irritating effects of gastroduodenal content on the distal esophagus [7]. Total (i.e. $360^{\circ}$ ) Nissen fundoplication (NF) is considered the most effective amongst these procedures [8]. According to many authors, the laparoscopic version of this procedure (LNF) represents a gold standard in patients qualified for surgical treatment due to both uncomplicated GERD and GERD associated with Barrett's metaplasia $[9,10]$.

Still, controversies related to the indications for anti-reflux surgery are frequently encountered. Most BE patients are qualified for LNF on the basis of the same criteria as subjects with uncomplicated GERD. These indications seem particularly justified in the case of younger patients, potentially exposed to a chronic injury of the esophageal mucosa. Perhaps the fact that more than one-third of BE patients do not experience reflux symptoms requiring chronic pharmacotherapy makes them one of the groups which can benefit from LNF. Due to the lack of pharmacological agents efficient against this condition, confirmed non-acidic reflux is deemed to constitute another important indication for surgical treatment.

The techniques of endoscopic ablation are used as an adjunct to surgical procedures in patients with dys- 
plasia of metaplastic epithelium. Such techniques, including argon plasma ablation, enable the re-epithelialization of the gastroesophageal junction [11].

\section{Aim}

The aim of this study was to perform a retrospective analysis of long-term treatment outcomes in patients with BE subjected to laparoscopic Nissen fundoplication.

\section{Material and methods}

A total of 168 laparoscopic Nissen fundoplications were performed in reflux disease patients of the Second Department of Surgery, Jagiellonian University Medical College, between January $1^{\text {st }}, 2001$ and April 30th, 2012. This group included $42 \mathrm{BE}$ patients, amongst them 30 men and 12 women. Mean age of enrolled patients was 49 years, and mean duration of followup amounted to 62 months (range: 3-94 months).

Endoscopic examination of the upper gastrointestinal tract was conducted in all patients during the preparatory period before surgery, and biopsy specimens were obtained from four esophageal quadrants to confirm the diagnosis. The biopsies were taken at $1 \mathrm{~cm}$ intervals at the level of the metaplastic segment, in accordance with the recommendations of the Montreal consensus (2006). Control endoscopy was performed 12 months after surgery. If persistent metaplastic lesions were observed, further control endoscopies were performed on an annual basis.

Initially, all the patients were treated conservatively (life style modification, dietary changes, PPIs) for at least one year. Mean duration of therapy was 2.5 years (range: 1-4 years).

Signs of dysplasia in the baseline specimens of gastroesophageal junction mucosa were observed in $20(47.6 \%)$ of the patients. This subgroup was subjected to preoperative argon plasma coagulation (APC) in order to eliminate dysplasia, since the lack of the latter was required to perform LNF. Endoscopic ablation was not performed in the remaining 22 (52.4\%) patients with no signs of dysplasia within the metaplastic epithelium.

During the surgery, diaphragmatic crura were sewn together in all the patients irrespective of the size of the hiatal hernia. The thoracic esophagus was mobilized up to $7 \mathrm{~cm}$ above the hiatal line. Both the diaphragmatic crura and the fundoplication wrap were sutured using non-absorbable stitches.
A complete circular metaplastic segment was revealed in 27 (64.3\%) patients, while 15 (35.7\%) patients presented with incomplete circular metaplasia. The classic (> $3 \mathrm{~cm}$ ) BE was observed in 25 (59.5\%) patients. Maximal extent of metaplasia in this group of patients was $5 \mathrm{~cm}$. The "short segment" type of BE was documented in $17(40.5 \%)$ individuals. On preoperative endoscopy, 38 (90.5\%) patients showed sliding hiatal hernia; 4 subjects (9.5\%) had mixed type of hiatal hernia.

One year after surgery, control biopsy specimens were obtained from the gastroesophageal junction of all the patients.

For the purpose of further analysis, the patients were divided into two groups based on the lack (group A) or presence of dysplasia (group B).

\section{Results}

None of the patients showed the development of esophageal adenocarcinoma during the follow-up period. Furthermore, no cases of dysplasia progression or de novo development of dysplasia were observed in the analyzed group. In 36 (85.7\%) subjects, laparoscopic Nissen fundoplication was reflected by the attenuation of reflux disease symptoms during the observation period. Thirty-three (78.6\%) patients reported transient swallowing disorders during the first 2 months after surgery. These complaints did not require hospitalization and resolved spontaneously in most cases. Nine (21.4\%) patients were treated successfully with oral hydroxyzine $(2 \times 25 \mathrm{mg})$.

In the first 12-24 months after surgery, complete regression of metaplasia was documented in 7 (31.8\%) patients from group $A$, and a reduction in the area of Barrett's metaplasia was observed in another 7 patients (31.8\%). The remaining 8 (36.4\%) patients did not show any endoscopically or histopathologically evident changes. The effects of anti-reflux therapy were observed in patients with no baseline signs of dysplasia, with gastric metaplasia $(n=3)$, and with complete intestinal metaplasia $(n=4)$; the so-called "short segment" type lesions were observed in all these patients. Throughout the period of this study, persistent planoepithelial re-epithelialization was observed in $14(70 \%)$ group B patients, i.e. in individuals with baseline dysplasia subjected to preoperative argon plasma ablation. In the remaining patients of this group, the developed changes of BE character were less advanced than at baseline. 


\section{Discussion}

Barrett's esophagus represents the most serious complication of gastroesophageal reflux disease, being an established precancerous lesion. While one can easily find the therapeutic recommendations regarding uncomplicated GERD in the available literature, the management of patients with glandular esophageal metaplasia still raises many questions.

Progression of Barrett's metaplasia towards esophageal adenocarcinoma results from chronic refluxate-induced injury of the esophageal mucosa. While $B E$ is a reversible state, persistent chronic inflammation promotes carcinogenesis [12]. Chemical composition of the refluxate seems to play an exceptionally important role in BE pathogenesis; specifically, the concentration of bile acids in reflux content is particularly correlated with the degree of esophageal mucosal injury [13].

It is widely accepted that controlling the signs of gastroesophageal reflux disease constitutes the principal therapeutic objective [2]. Achieving this therapeutic effect requires normalization of $\mathrm{pH}$ at the esophageal-gastric junction; this goal is mostly achieved with proton pump inhibitors (PPI). This approach attenuates the signs of reflux disease by reducing the intensity of inflammatory lesions in the distal esophagus. However, a number of authors point to the limited value of such an approach in some patients. Mikes and Gerson observed persistent acidic reflux on $\mathrm{pH}$-metric examination in $50 \%$ of asymptomatic patients treated with PPIs [14]. Also, the evidence of negative consequences associated with persistent hypergastrinemia should not be underestimated.

Anti-reflux surgery, optimally performed laparoscopically, remains an alternative therapeutic approach as the only modality of treating the cause in patients with dysfunction of the lower esophageal sphincter. Such treatment enables restoration of the physiological barrier reducing exposure of the esophagus to reflux content.

Reduction of the area of glandular metaplasia constitutes the principal component of adjunctive treatment in BE patients. Most commonly, ablation techniques are recommended in patients with associated dysplasia. The most popular methods include photodynamic technique, ablation with radiofrequency waves, and argon plasma ablation.
All the above-mentioned facts unambiguously substantiate administration of chronic anti-secretory therapy in the majority of BE patients. Currently, most authors recommend basing the therapy on PPIs, although a unified therapeutic protocol has not been developed thus far. The most frequently postulated protocol is based on over-the-standard dosage of PPIs; however, this approach is associated with established, more severe, and prolonged course of reflux disease in $\mathrm{BE}$ patients as compared to the group with uncomplicated GERD. The available literature contains reports suggesting the possibility of complete remission of glandular metaplasia in patients treated solely with PPIs; nevertheless, authors assign varying significance to these findings. In a double-blinded randomized study, Peters observed a complete remission of Barrett's metaplasia in $8 \%$ of patients subjected to 24 months of continuous therapy with $80 \mathrm{mg}$ of omeprazole [15]. Simultaneously, there were no cases of complete regression of metaplastic lesions documented in a ranitidine-treated group. In the opinion of the majority of authors, chronic administration of proton pump inhibitors can lead only to reduction of the metaplastic area with potential development of islets lined with normal squamous epithelium [16, 17]. However, according to some opinions, the residual foci of metaplastic epithelium can be localized deeper than the newly formed islets, raising the question about the effectiveness of the aforementioned therapeutic approach.

The above-mentioned data suggest that pharmacotherapy alone is not reflected by regression of glandular metaplasia in the majority of BE patients and does not enable planoepithelial re-epithelialization. There is increasing evidence of the necessity of implementing one of the ablation techniques at the early stage of treatment in order to remove the metaplastic foci frequently associated with dysplasia. Combining endoscopic techniques with anti-secretory therapy is also reflected by persistent planoepithelial re-epithelialization [18]. In our study, the combination of argon plasma ablation with laparoscopic Nissen fundoplication enabled persistent elimination of Barrett's metaplasia in approximately $70 \%$ of the patients. However, the available literature lacks unambiguous confirmation of the protective effect of such an approach with regards to the risk of esophageal adenocarcinoma; this necessitates further research on the problem in question $[19,20]$. 
Anti-reflux surgery has become a common component of treatment, particularly due to the popularization of the laparoscopic approach. Pharmacotherapy enables a transient normalization of $\mathrm{pH}$ at the esophageal-gastric junction, but does not modulate the efficiency of the physiological anti-reflux barrier. Patients subjected to argon plasma ablation of metaplastic mucosa require long-term elimination of the harmful effect of stomach-regurgitated content in order to establish the therapeutic outcome. Nissen fundoplication is currently postulated as the most efficient modality of reflux treatment. Many authors consider the laparoscopic form of this procedure as a gold standard of GERD therapy. Surgical treatment seems particularly substantiated in BE patients, particularly in those lacking reflux symptoms. According to Sharma, surgical treatment plays a vital role in the management of BE patients, as well as in the prevention of BE-induced esophageal adenocarcinoma [21]. The outcomes of laparoscopic anti-reflux surgery proved excellent with regards to the attenuation of reflux symptoms. In Parill's study, the control of GERD symptoms was achieved in $91 \%$ of operated patients [22]. In Eubanks' analysis, mean 43-month postsurgical follow-up revealed a $95 \%$ reduction of reflux disorders [23]. In our study, the typical GERD-related complaints resolved in $86 \%$ of patients analyzed during the period of the experiment.

According to numerous authors, there is a predominance of alkaline component of the reflux in patients with $B E[24,25]$. The authors of previous reports emphasize that the degree of esophageal mucosal exposure to bile content in BE patients is similar to that observed in individuals subjected to partial gastric resection. These facts point to limited effectiveness of pharmacotherapy in this group of patients. Consequently, surgical restoration of the anti-reflux barrier should be markedly more effective in the normalization of this type of reflux. In Parilla's study, limited exposure to bile content was observed in $92 \%$ of the patients subjected to the anti-reflux procedure and in only $25 \%$ of individuals treated with PPI [22]. Consequently, it could be expected that the outcomes regarding reduction of the metaplastic area and the prevention of dysplasia and development of malignancy would be markedly better in surgically treated patients than in those in whom ablation techniques were combined with anti-secretory pharmacotherapy.
Current research suggests the possibility of complete regression of metaplasia after surgical correction of an inefficient anti-reflux barrier. This phenomenon was observed in 4 out of 10 surgically treated patients included in Brand's study, the findings of which were published in 1980 [26]. In Oelschlager's report, during the mean 40-month follow-up period, the complete remission of metaplasia was revealed in $33 \%$ of 106 cases of laparoscopically operated patients with short BE. The results of previous analyses suggest that such an approach can be effective in no more than $20 \%$ of patients with classic BE. Also, reports suggesting the possibility of regression of $B E-$ related dysplasia can be found in the available literature [27-29]. The results of these studies suggest that complete regression of mild dysplasia can be obtained in even up to $70 \%$ of the patients. Complete regression of glandular metaplasia was documented in seven of the analyzed patients with short BE.

The prevailing opinion indicates that Nissen fundoplication, as a result of high effectiveness, represents the method of choice in the treatment of BE in the case of patients who were qualified for surgery. However, such a consistent attitude is lacking with regards to the details of its modifications. Early experiences with LNF were limited to mobilization of the esophagus solely at the level of the esophageal hiatus. Currently, according to the recommendations of acknowledged authorities, the threshold of mobilization should be placed much higher (Photo 2). However, there are some discrepancies with regards to the level of esophageal mobilization in the mediastinum. According to Dallemagne, the esophagus should be

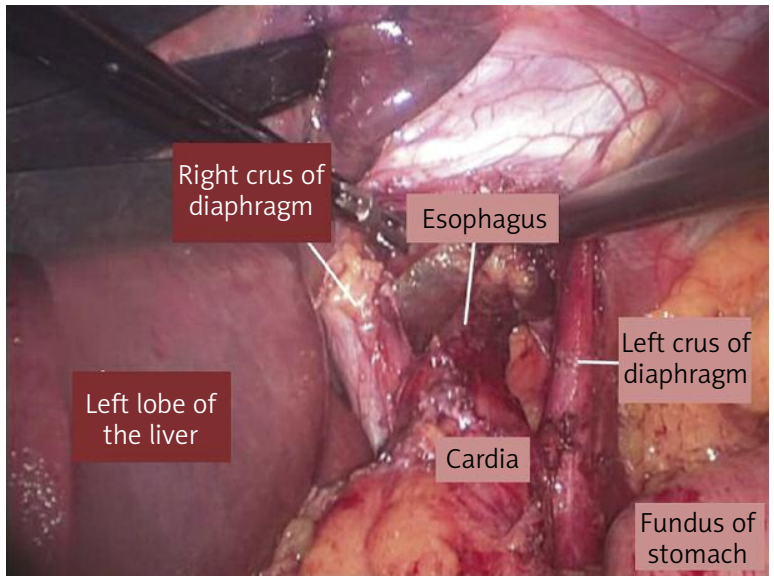

Photo 2. Laparoscopic Nissen fundoplication intraoperative view during preparation of the esophagus within the mediastinum 
mobilized up to $5-7 \mathrm{~cm}$ above the esophageal hiatus [30]. In contrast, Swanstrom defines this threshold at the level of inferior pulmonary veins [31]. Such a maneuver enables the restoration of a sufficiently long abdominal section of the esophagus, which seems to be one of the most important components of the anti-reflux barrier. Furthermore, high mobilization of the esophagus along with tighter than previously postulated suturing of the diaphragmatic crura prevents translocation of the fundoplication wrap into the mediastinum. According to Hunter et al., this complication represents the most frequently reported reason for re-operation in this group of patients [32].

Many surgeons believe that the diaphragmatic crura should be sutured together only in the case of evident signs of hiatal hernia. However, according to increasing evidence, the tight binding of the crura in all patients subjected to fundoplication constitutes one of the main determinants of a successful outcome. According to recently published opinions, such an approach does not increase the risk of dysphagia symptoms. Transient problems with swallowing, which were also observed in our patients, probably result from transient swelling of the esophagus at the level of the fundoplication wrap. Additionally, normal swallowing can be impaired in some patients as a result of stress, as confirmed by high effectiveness of sedative agents. However, most of such complaints resolve spontaneously; intervention is required rarely, and most commonly is limited to endoscopic balloon dilatation.

\section{References}

1. Caygill CP, Watson A, Reed PI, Hill MJ. UK National Barrett's Oesophagus Registry (UKBOR) and the 27 Participating Centres. Characteristics and regional variations of patients with Barrett's oesophagus in the UK. Eur J Gastroenterol Hepatol 2003; 15: 1217-22.

2. Sampliner RE. Practice Parameters Committee of the American College of Gastroenterology. Updated guidelines for the diagnosis, surveillance, and therapy of Barrett's esophagus. Am J Gastroenterol 2002; 97: 1888-95.

3. Gutschow CA, Schröder W, Hölscher AH. Barrett's esophagus: what is the poison - alkaline, biliary or acidic reflux? Dis Esophagus 2002; 15: 5-9.

4. Kauer WK, Stein HJ. Role of acid and bile in the genesis of Barrett's esophagus. Chest Surg Clin N Am 2002; 12: 39-45.

5. Nason KS, Farrow DC, Haigh G, et al. Gastric fluid bile concentrations and risk of Barrett's esophagus. Interact Cardiovasc Thorac Surg 2007; 6: 304-7.

6. Hongo M. Barrett's oesophagus and carcinoma in Japan. Aliment Pharmacol Ther 2004; 20 Suppl 8: 50-4.
7. Tarnowski W, Kiciak A, Borycka-Kiciak K, et al. Laparoscopic fundoplication improves oesophageal motility - a prospective study. Videosurgery Miniinv 2011; 6: 73-83.

8. Szyca R, Leksowski K, Cornelia de Lange syndrome - characteristics and laparoscopic treatment modalities of reflux based on own material. Videosurgery Miniinv 2011; 6: 173-7.

9. Wróblewski T, Skalski M, Ziarkiewicz-Wróblewska B, et al. New antireflux surgical technique in GERD treatment. Videosurgery Miniinv 2007; 2: 139-44.

10. Wróblewski T, Skalski M, Ziarkiewicz-Wróblewska B, et al. Progress in surgical treatment of reflux disease. Videosurgery Miniinv 2006; 1: 121-4.

11. Migaczewski M, Budzyński A, Rembiasz K. Argon plasma coagulation (APC) for treatment of Barrett's oesophagus. Videosurgery Miniinv 2009; 4: 102-9.

12. Lagergren J, Bergstrom R, Lindgren A, et al. Symptomatic gastroesophageal reflux as a risk factor for esophageal adenocarcinoma. N Engl J Med 1999; 340: 825-31.

13. DeMeester SR. Adenocarcinoma of the esophagus and cardia: a review of the disease and its treatment. Ann Surg Oncol 2006; 13: $12-30$.

14. Milkes D, Gerson LB, Triadafilopoulos G. Complete elimination of reflux symptoms does not guarantee normalization of intraesophageal and intragastric $\mathrm{pH}$ in patients with gastroesophageal reflux disease (GERD). Am J Gastroenterol 2004; 99: 991-6.

15. Peters FT, Ganesh S, Kuipers EJ, et al. Endoscopic regression of Barrett's oesophagus during omeprazole treatment: a randomised double blind study. Gut 1999; 45: 489-94.

16. Chaves P, Pereira AD, Cruz C, et al. Recurrent columnar-lined esophageal segments: study of the phenotypic characteristics using intestinal markers. Dis Esophagus 2002; 15: 282-6.

17. Ertan A, Younes M. Barrett's esophagus. Dig Dis Sci 2000; 45 : 1670-3.

18. Kahaleh M, Van Laethem JL, Nagy N, et al. Long-term follow-up and factors predictive of recurrence in Barrett's esophagus treated by argon plasma coagulation and acid suppression. Endoscopy 2002; 34: 950-5.

19. Wang KK, Sampliner RE. Practice Parameters Committee of the American College of Gastroenterology, Updated guidelines 2008 for the diagnosis, surveillance and therapy of Barrett's esophagus. Am J Gastroenterol 2008; 103: 788-97.

20. Schulz H, Miehlke S, Antos D, et al. Ablation of Barrett's epithelium by endoscopic argon plasma coagulation in combination with high-dose omeprazole. Gastrointest Endosc 2000; 51 : 659-63.

21. Sharma P, Sampliner R. Barrett's esophagus, and esophageal adenocarcinoma. Blackwell Publishing 2006.

22. Parrilla P, Martínez de Haro LF, Ortiz A, et al. Long-term results of a randomized prospective study comparing medical and surgical treatment of Barrett's esophagus. Ann Surg 2003; 237: 291-8.

23. Eubanks TR, Omelanczuk P, Richards C, et al. Outcomes of laparoscopic antireflux procedures. Am J Surg 2000; 179: 391-5.

24. Stein HJ, Kauer WK, Feussner H, Siewert JR. Bile reflux in benign and malignant Barrett's esophagus: effect of medical acid suppression and Nissen fundoplication. J Gastrointest Surg 1998; 2: 333-41. 
25. Champion G, Richter JE, Vaezi MF, et al. Duodenogastroesophageal reflux: relationship to $\mathrm{pH}$ and importance in Barrett's esophagus. Gastroenterology 1994; 107: 747-54.

26. Brand DL, Ylvisaker JT, Gelfand M, Pope CE 2nd. Regression of columnar esophageal (Barrett's) epithelium after anti-reflux surgery. N Engl J Med 1980; 302: 844-8.

27. Farrell TM, Smith CD, Metreveli RE, et al. Fundoplication provides effective and durable symptom relief in patients with Barrett's esophagus. Am J Surg 1999; 178: 18-21.

28. Low DE, Levine DS, Dail DH, Kozarek RA. Histological and anatomic changes in Barrett's esophagus after antireflux surgery. Am J Gastroenterol 1999; 94: 80-5.

29. DeMeester SR, Campos GM, DeMeester TR, et al. The impact of an antireflux procedure on intestinal metaplasia of the cardia. Ann Surg 1998; 228: 547-56.

30. Dallemagne B, Weerts J, Markiewicz S, et al. Clinical results of laparoscopic fundoplication at ten years after surgery. Surg Endosc 2006; 20: 159-65.

31. Swanstrom LL. Management of patients with gastroesophageal reflux disease and esophageal or gastric dysmotility. J Gastrointest Surg 2001; 5: 448-50.

32. Hunter JG, Smith CD, Branum GD, et al. Laparoscopic fundoplication failures: patterns of failure and response to fundoplication revision. Ann Surg 1999; 230: 595-604.

Received: 14.10.2012, revised: 15.11.2012, accepted: 29.11.2012. 